\title{
BMJ Open Association between muscle strength and health-related quality of life in a Chinese rural elderly population: a cross-sectional study
}

\author{
Shanshan Yang, ${ }^{1,2}$ Tianzhi Li, ${ }^{3}$ Hongbing Yang, ${ }^{4}$ Jianhua Wang, ${ }^{1}$ Miao Liu, ${ }^{1}$ \\ Shengshu Wang, ${ }^{1}$ Yao He, ${ }^{1}$ Bin Jiang ${ }^{5}$
}

To cite: Yang S, Li T, Yang $\mathrm{H}$, et al. Association between muscle strength and health-related quality of life in a Chinese rural elderly population: a crosssectional study. BMJ Open 2020;10:e026560. doi:10.1136/ bmjopen-2018-026560

- Prepublication history and additional material for this paper are available online. To view these files, please visit the journal online(http://dx.doi. org/10.1136/bmjopen-2018026560).

SY and TL contributed equally.

Received 13 September 2018 Revised 19 June 2019 Accepted 26 June 2019

Check for updates

(C) Author(s) (or their employer(s)) 2020. Re-use permitted under CC BY-NC. No commercial re-use. See rights and permissions. Published by BMJ.

For numbered affiliations see end of article.

Correspondence to Hongbing Yang; yanghongbing7953@126.com and Dr. Bin Jiang; jiangbin301@126.com

\section{ABSTRACT}

Objective To investigate the relationship between upper and lower limb muscle strength and the healthrelated quality of life (HRQOL) of a Chinese rural, elderly population.

Design A population-based, cross-sectional study. Setting Miyun, Beijing, China.

Participants The participants of this study were 2083 (834 men and 1249 women) older adults from a rural area, (average age of $\geq 60$ years), living in Miyun county, located on the outskirts of Beijing. Data were collected between May and October 2014. Handgrip strength and timed up and go tests (TUGT) were conducted to measure the muscle strength of their upper and lower limbs, respectively. The Euro Quality of Life (Euroqol) (EQ-5D)Visual Analogue Scale was used to evaluate participants' HRQoL.

Results A significant association between handgrip strength and the EQ-5D index ( $\beta=0.015$ per SD, $95 \% \mathrm{Cl}$ : 0.008 to $0.023, p<0.001$ ) was discovered, following adjustments. The association between handgrip strength and the EQ-5D index in the $\geq 80$ years group was found to be stronger than that of the 60-79 years group, following adjustment ( $\beta$ per SD: 0.013 vs 0.035 ). Similar results were observed when comparing the non-chronic disease group, in terms of TUGT time, against those with chronic diseases.

Conclusions There was a significant relationship between muscle strength (measured via handgrip strength and TUGT time) and HRQoL (measured via EQ-5D index and VAS score) in the Chinese rural elderly population. Furthermore, this relationship was stronger in the older population (aged $\geq 80$ years), and in those participants diagnosed with chronic diseases.

\section{INTRODUCTION}

\section{Background}

The proportion of people aged $\geq 60$ years is rapidly increasing internationally, and is expected to further increase from $12 \%$ in 2013 , to $21 \%$ in $2050^{1}$. China is currently facing the additional challenge of a dramatically ageing population. In 2013, there were more than 200 million elderly people living
Strengths and limitations of this study

Only a few studies have been conducted on the association between muscle strength and the health-related quality of life ( $\mathrm{HRQ} \mathrm{L}$ ) for the Chinese rural elderly population, especially among those living in rural areas; whereas this study used a large sample population-based cross-sectional design in order to research this association within this demographic.

- In addition to upper limb muscle strength, lower limb muscle strength was also included in this study, with the association between muscle strength and HRQoL being analysed between different subgroups.

- Our sample may not be completely representative of the entire Chinese rural elderly population, as our participants resided in an area on the outskirts of Beijing, which is under relatively high economic conditions.

- Due to the inherent bias of cross-sectional studies, we could not conclude if there was a causal correlation between muscle strength and HRQOL in the participants.

within China, with this number expected to increase to 437 million by $2051 .{ }^{23}$ With the increased acceleration of the ageing process, disabilities and a low health-related quality of life (HRQoL) have become increasingly prominent among the elderly population in China. HRQoL is a comprehensive reflection of various health-related factors, such as physical health, psychological status, degree of independence, social relationships and environmental factors. ${ }^{4}$ HRQoL has been extensively incorporated as a primary or secondary outcome in many studies. ${ }^{5}$ Previous studies have shown that elderly individuals who were physically active generally possessed a higher HRQoL than those who were more inactive ${ }^{6}$ with others uncovering that muscle strength was related to factors like physical fitness, disability and mortality. ${ }^{7-9}$ However, studies on the association between muscle 
strength and HRQoL are limited, with only a relative few involving older adults in the community and using handgrip strength (without evaluating lower limb strength) as a marker of muscle strength. ${ }^{10}{ }^{11}$ A recent study found that the muscle strength of an individual's lower limbs, which may influence their balance skills, may be more influential than handgrip strength in terms of HRQoL in the pre-frail and frail, community-dwelling older adults in Vienna, Austria. ${ }^{10}$ In addition, due to differing cultural backgrounds and the social structures from Western countries, the relationship between muscle strength and HRQoL may be different within a Chinese elderly population. To our knowledge, only a relatively few studies have been conducted on the association between muscle strength and HRQoL for a Chinese elderly population. As $73.7 \%$ of the Chinese elderly population live in rural areas, ${ }^{311}$ more attention should be paid to factors influencing their HRQoL.

\section{Objectives}

Thus, in this study, we aimed to investigate the characteristics of the HRQoL of a Chinese rural elderly population, as well as the association between muscle strength and their overall HRQoL.

\section{Design and methods \\ Study sample}

A population-based cross-sectional survey was conducted from May to October 2014, involving an elderly population (age $\geq 60$ years) who lived in Miyun county, located on the outskirts of Beijing, as the study participants. In 2014, there were 17 towns (including 34 communities and 330 villages) with 43318 elderly residents. Therefore, a randomised cluster sampling method was used to select 2 of these 17 towns (one from a set of nine towns including both communities and village committees, and one from eight towns including only village committees). All households with elderly residents $(n=2589)$ within the two towns were selected and, from each household, one elderly resident was randomly selected as a participant. A total of 2397 participants (967 men and 1430 women) completed the survey, with a response rate of $92.6 \%$. After excluding 314 participants with missing muscle strength data, a total of 2083 participants (834 men and 1249 women) were included in our study.

Data regarding participants' gender, age, occupational status, nationality, alcohol consumption and smoking statuses, physical activity level and chronic disease status (including coronary heart disease (CHD), stroke and type 2 diabetes mellitus (T2DM)) were collected by interviewers and physicians trained at the People's Liberation Army (PLA) General Hospital. In some studies, participants aged 80 years and over were referred to as 'old-old,' whereas those aged 60-79 years were called 'young-old'. ${ }^{12}$ In China, individuals aged 80 years and above receive an 'old age' allowance from the government and, therefore, in this study we divided the age groups into those aged $60-79$ and $80+$ years, respectively.

\section{Measurements}

\section{Muscle strength}

Participants' handgrip strength was used as an indicator of their upper limb muscle strength level, and was measured using a Jamar hand dynamometer (Sammons Preston, Bolingbrook, Illinois, USA) by trained physicians from the PLA General Hospital (expressed in kilograms). First, the width of the device's handle was adjusted to ensure that the middle phalanx of each participant's hand rested on the inner handle. Thereafter, the participants were asked to hold the dynamometer using their hands, with their arm parallel to their body, in an upright position (squeezing the arm against the body was not allowed). The participants were then asked to perform three test trials on each hand, with the best score being used for the final analysis. The original values were recorded without adjusting for participant's individual sex or body mass index (BMI).

The timed up and go test (TUGT) time of each participant was used to measure their lower limb muscle strength, and was conducted using an armchair and a stopwatch, ${ }^{1314}$ by trained physicians from the PLA General Hospital (expressed in seconds). The participants were asked to sit on the provided armchair, with their hands on the chair's arms, and to stand up after hearing a password from the physician (whereby timing began on the stopwatch), and to walk $3 \mathrm{~m}$ forward and back before sitting down again (timing ends). The original values were recorded without adjusting for sex or BMI. A shorter time indicated better lower limb muscle strength and balance capacity. ${ }^{15}$

\section{Health-related quality of life}

The EQ-5D-VAS ${ }^{12}$ was used in order to measure participants' overall HRQoL. The EQ-5D is a validated and extensively used general health questionnaire that covers five health domains (mobility, self-care, usual activities, pain/discomfort and anxiety/depression). The visual Analogue Scale (VAS) is a self-rating tool for measuring a person's health status using a $20 \mathrm{~cm}$ vertical scale $(100$, at the top, indicating the best health status, and 0 , at the bottom, indicating the worst). Trained interviewers collected the questionnaire information gained by the EQ-5D and VAS, with the health index being calculated using the Japanese population-based time trade-off (TTO) model. A previous study uncovered that the Japanese TTO model is, currently, the most suitable tool for studying Chinese individuals. ${ }^{16}$

\section{Covariables}

In this study, the chronic diseases considered included three common illnesses found among the Chinese population: CHD, stroke and T2DM. CHD and stroke were defined using the WHO MONICA's criteria, ${ }^{17}$ with T2DM being defined via the American Diabetes Association's criteria. ${ }^{18}$ The participants' statuses of CHD, stroke and T2DM were collected by trained interviewers and physicians from the PLA General Hospital. These medical 
conditions were self-reported by the respondents, and were then verified by professional doctors who checked their medical records.

Alcoholics were defined as regular drinkers who had consumed alcohol for almost every day for more than half a year. A former alcohol user was defined as an individual who drank alcohol daily, for at least 6 months during their lives, but who had not been engaging in this behaviour at the time of the survey. A current smoker was defined as an individual who regularly smoked tobacco products, at the time of the survey. A former smoker was defined as an individual who had smoked daily for at least 6 months during their lives, but had not been using any tobacco products at the time of the survey. ${ }^{19}{ }^{20}$ BMI was calculated as follows: weight $(\mathrm{kg}) /$ standing height $(\mathrm{m})^{2}$, with participants' weight and height being measured by trained nurses. Physical activity levels were self-reported and classified as follows: (1) never, (2) $<5$ hours/week, (3) 5-7.9 hours/week and (4) $\geq 8$ hours/week. This information was collected by trained interviewers, who spoke with the participants face to face. Educational levels were self-reported and classified as illiterate, primary school, middle school, college and above. Marital statuses were classified as married, living together, single, divorced or widowed. Finally, occupational statuses were classified as white collar, light physical labour and hard physical labour.

\section{Statistical analysis}

SPSS V.19.0 and R V.3.5.2 were used for the data analysis. The significance level for all tests was set at a two-tailed $\alpha$ value of 0.05 . The differences in the means and proportions were evaluated using Student's t-test and a $\mathrm{X}^{2}$ test, respectively. Linear regression models were used to identify the associations between handgrip strength/ TUGT time and the EQ-5D/VAS findings, with handgrip strength/TUGT time being included as continuous variables (per unit, per 10\%, and per SD). Restricted cubic regression splines were used in order to model the functional forms of the non-linear effects of handgrip strength/TUGT time and EQ-5D.

\section{Patient and public involvement}

No patients were involved in this study.

\section{RESULTS}

A total of 2083 (834 men and 1249 women) elderly rural residents were included in our study. Their average age was $69.4 \pm 6.9$ years (range: $60-95$ years). The average EQ-5D index was $0.93 \pm 0.13$ (range: -0.11 to 1.00 ), with the average VAS score being $74.38 \pm 14.89$ (range: $0-100$ ). The average EQ-5D indices of the participants with and without chronic diseases were $0.92 \pm 0.14$ and $0.94 \pm 0.12$, respectively. The coefficient of the EQ-5D and VAS was 0.538 for our sample $(p<0.05)$. The general characteristics (ie, age, sex, nationality, educational level, marital status, occupational status, smoking status, alcohol drinking status, chronic disease status, BMI, handgrip strength, TUGT time and EQ-5D-VAS findings) of the participants are shown in table 1 . Compared with the 60-79-year-old group, the $\geq 80$-year-old group consisted of individuals with the following characteristics: more physical labourers, lower educational levels, lower BMIs, lower handgrip strengths, longer TUGT times, lower EQ-5D indices and VAS scores (EQ-5D index: 0.94 \pm 0.12 vs $0.89 \pm 0.17$ and VAS score: $74.73 \pm 14.70$ vs $71.61 \pm 16.10$; $\mathrm{p}<0.05)$. It also included fewer married individuals, fewer people living together, fewer smokers and fewer alcohol drinkers (table 1).

In the linear regression analysis, we observed a significant association between participants' handgrip strength and their EQ-5D index ( $\beta=0.015$ per SD, $95 \%$ CI: 0.008 to $0.023, \mathrm{p}<0.001$ ), after adjusting for age, sex, nationality, educational level, occupational status, marital status, smoking status, alcohol drinking status, BMI, physical activity level, CHD, stroke and T2DM. We also observed a significant, negative relationship between the participants' TUGT time and EQ-5D index following a similar adjustment ( $\beta=-0.029$ per SD, 95\% CI: $-0.023-0.022$, $\mathrm{p}<0.001)$. When handgrip strength and TUGT time were analysed as continuous variables (per unit or per 10\%), the results remained consistent (all $\mathrm{p}<0.05$, table 2). Similar associations were observed between handgrip strength and VAS scores, as well as between TUGT times and VAS scores, following adjustment (table 3).

In addition, the association between handgrip strength and EQ-5D indices in the $\geq 80$ year-old group was stronger, following adjustment, than that in the 60-79year-old group ( $\beta$ per SD: 0.013 vs 0.035 , table 4 ). Similar results were observed in the relationship between TUGT time, EQ-5D index ( $\beta$ per SD: -0.026 vs -0.042 , table 4 ) and VAS score (supplementary table $\mathrm{S} 1$ ). The association between handgrip strength and EQ-5D index in those diagnosed with chronic diseases was stronger, after adjusting for their age, sex, nationality, educational level, occupation, marital status, smoking status, alcohol drinking status, BMI and physical activity level, when compared with those without chronic diseases ( $\beta$ per SD: 0.012 vs 0.022 , table 5). A similar result was observed in the relationship between TUGT time and EQ-5D index ( $\beta$ per SD: -0.026 vs -0.033 , table 5). Furthermore, the association between handgrip strength and participants' VAS scores was no longer significant following adjustment for those without chronic diseases, but was still significant after adjustment in those with chronic diseases ( $\beta=1.561$ per $\mathrm{SD}, 95 \% \mathrm{CI}$ : 0.235 to $2.887, \mathrm{p}=0.021$, supplementary table $\mathrm{S} 2$ ).

There were 1553 individuals (including 1409 aged $60-79$ years old and 144 aged $\geq 80$ years old; 601 with chronic diseases and 952 without) whose scores showed no problem in any dimension of the EQ-5D (recorded as a score of 1 ). We divided the sample into two groups (those scoring 1 vs those scoring less than 1 ), and conducted the logistic regression. The results demonstrated that, when compared with individuals with lower scores, people scoring higher for every $1 \mathrm{~kg}$ interval of handgrip strength 
Table 1 Baseline characteristics of the participants

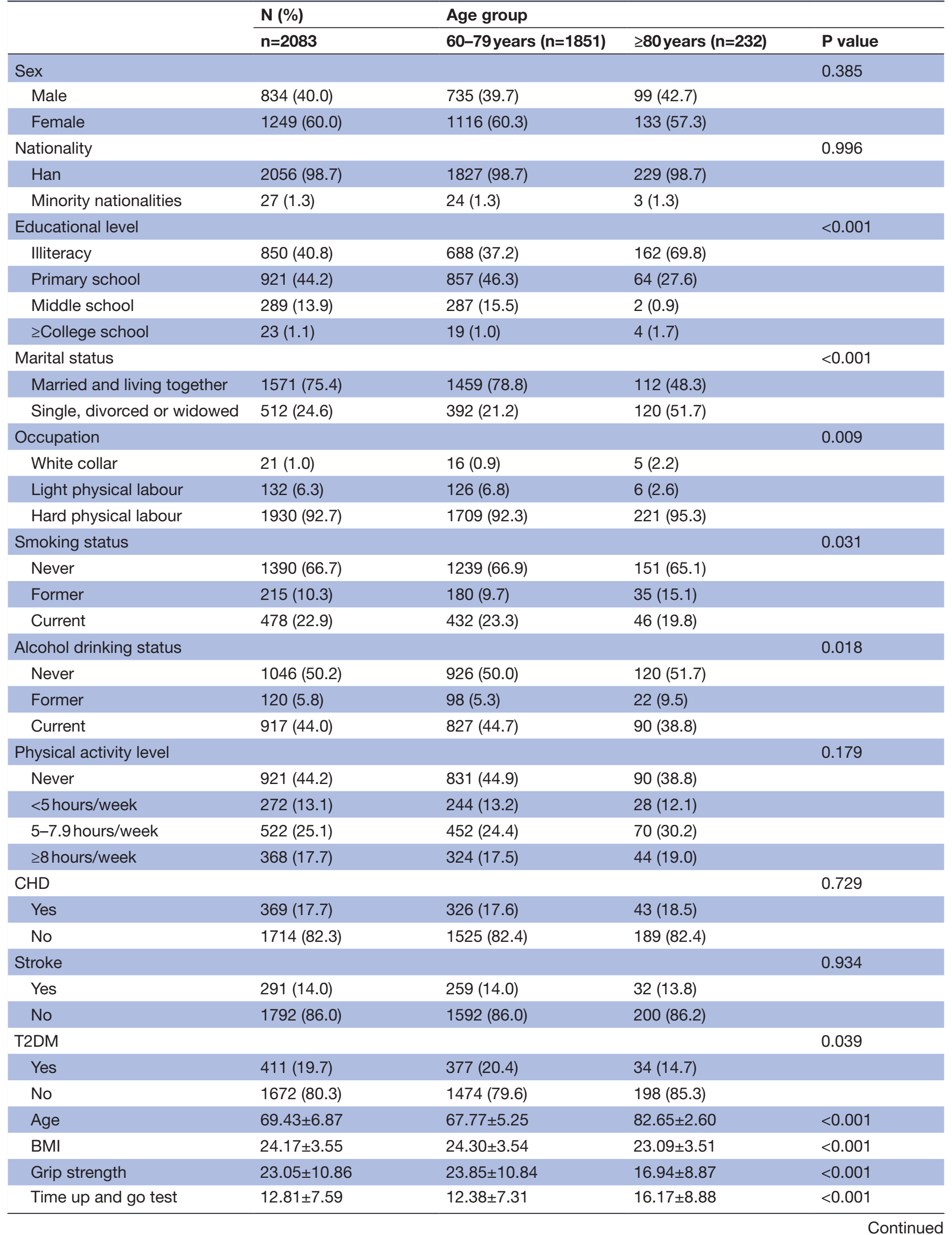




\begin{tabular}{|c|c|c|c|c|}
\hline & \multirow{2}{*}{$\begin{array}{l}N(\%) \\
n=2083\end{array}$} & \multicolumn{3}{|l|}{ Age group } \\
\hline & & $60-79$ years $(n=1851)$ & $\geq 80$ years $(n=232)$ & $P$ value \\
\hline$E Q-5 D$ & $0.93 \pm 0.13$ & $0.94 \pm 0.12$ & $0.89 \pm 0.17$ & $<0.001$ \\
\hline
\end{tabular}

BMI, body mass index; CHD, coronary heart disease; T2DM, type 2 diabetes mellitus; VAS, Visual Analogue Scale.

had a greater chance to receive a score of 1 , showing no problem in any dimension of the EQ-5D $(\mathrm{OR}=1.017$, 95\% CI: 1.004 to 1.030), after adjusting for age, sex, nationality, educational level, occupation, marital status, smoking status, alcohol drinking status, BMI, physical activity level, CHD, stroke and T2DM. When compared with individuals with shorter TUTG times, those with longer times per $1 \mathrm{~s}$ interval possessed lower chances to receive a score of 1 with no problem in any dimension of the EQ-5D (OR=0.971, 95\% CI: 0.958 to 0.983$)$, following adjustments (supplementary table S3). We conducted the analysis for the 60-79 and $\geq 80$-year-old groups, with and without chronic diseases, respectively, and the results were similar to the linear regression analysis (supplementary table S3).

Furthermore, in order to model the functional forms of the non-linear effects of handgrip strength, TUGT times and EQ-5D scores of 1, a restricted cubic regression splines in $\mathrm{R}$ was done, with the results shown in figure 1 . This non-linear test uncovered that the association between handgrip strength and the OR of EQ-5D scores of 1 was linear $(p=0.7209)$, whereas the association between TUGT times and the OR of EQ-5D score of 1 was non-linear $(\mathrm{p}<0.001)$.

\section{DISCUSSION}

In this study, we observed a significant association between muscle strength (measured via handgrip strength and TUGT times) and HRQoL (measured via the EQ-5D index and VAS scores) in a Chinese rural elderly population. The results demonstrated that the functional forms of handgrip strength and TUGT time were different. The associations were stronger for the $\geq 80$-year-old group and participants with chronic diseases, than for the 60-79-year-old group and those without chronic diseases.

Participants' health indices were calculated using the Japanese population-based TTO model. The health measures of differing TTO models reflects subjective experiences based on specific cultural and social backgrounds and, up until now, no such model for the Chinese population existed. However, the Japanese population shares a similar cultural and social background with their Chinese counterparts. In particular, when compared against English and American TTO models, the Japanese version has a higher separating capacity for stroke, CHD and hypertension, as found in the 65-79year old Chinese population. ${ }^{16}$ The coefficient of the EQ-5D and VAS measures in our sample was 0.538 , which is similar to the fifth National Health Service Survey of China, conducted in $2013,{ }^{21}$ and higher than that found in the UK, ${ }^{22}$ where such discrepancies may be due to differences in the social culture and structure. Another previous study, ${ }^{23}$ on the HRQoL of a Chinese western rural population, indicated that the VAS score was 67.2. However, the EQ-5D index and SD of the VAS score were not reported. For the difference due to territory, the VAS score of our study population was higher than that of the western rural population. Our VAS scores were similar with those of a study conducted in an urban district (Dongcheng) in Beijing. ${ }^{24}$ This study reported that the VAS score of their participants was $78.3 \pm 16.2$ and was $70.7 \pm 16.8$ for those with chronic conditions. However, the EQ-5D index, measured through a TTO convertible calculation, was not reported by this study.

A study among elderly individuals with pre-diabetes ${ }^{6}$ showed that the overall HRQoL of elderly individuals is poor in rural China, and that it is associated with a person's physical activity. A study conducted with a Korean population ${ }^{25}$ showed that all types of exercises (resistance, flexibility and walking) demonstrated higher HRQoL scores when compared with people engaging in no exercise at all. A previous study uncovered that, for older people living in the Netherlands ( $>85$ years old) ${ }^{26}$ a lower handgrip strength was a predictor of a decline in their performance in activities around daily living and cognition $(\mathrm{p}<0.001)$. A study on 65 -year-old individuals who had experienced falls ${ }^{27}$ found that the TUGT time was associated with recurrent falls and overall HRQoL. The aforementioned studies suggest two possible ways in which muscle strength could influence a person's HRQoL-through a decreased athletic performance and an increased risk of falls. Similar to our results, a study on men and women in the UK, aged 59-73 years, ${ }^{28}$ found that a lower grip strength is associated with reduced HRQoL, after adjusting for age, size, physical activity level and chronic conditions. In this study, we discovered that TUGT time was also associated with the HRQoL of a Chinese rural elderly population, following adjustment.

This study had several limitations. As the information on the EQ-5D index was self- reported, bias caused by individual, subjective differences cannot be fully ruled out. However, the information was confirmed with the participants and their relatives in order to ensure the data's accuracy. Second, our sample may not be completely 

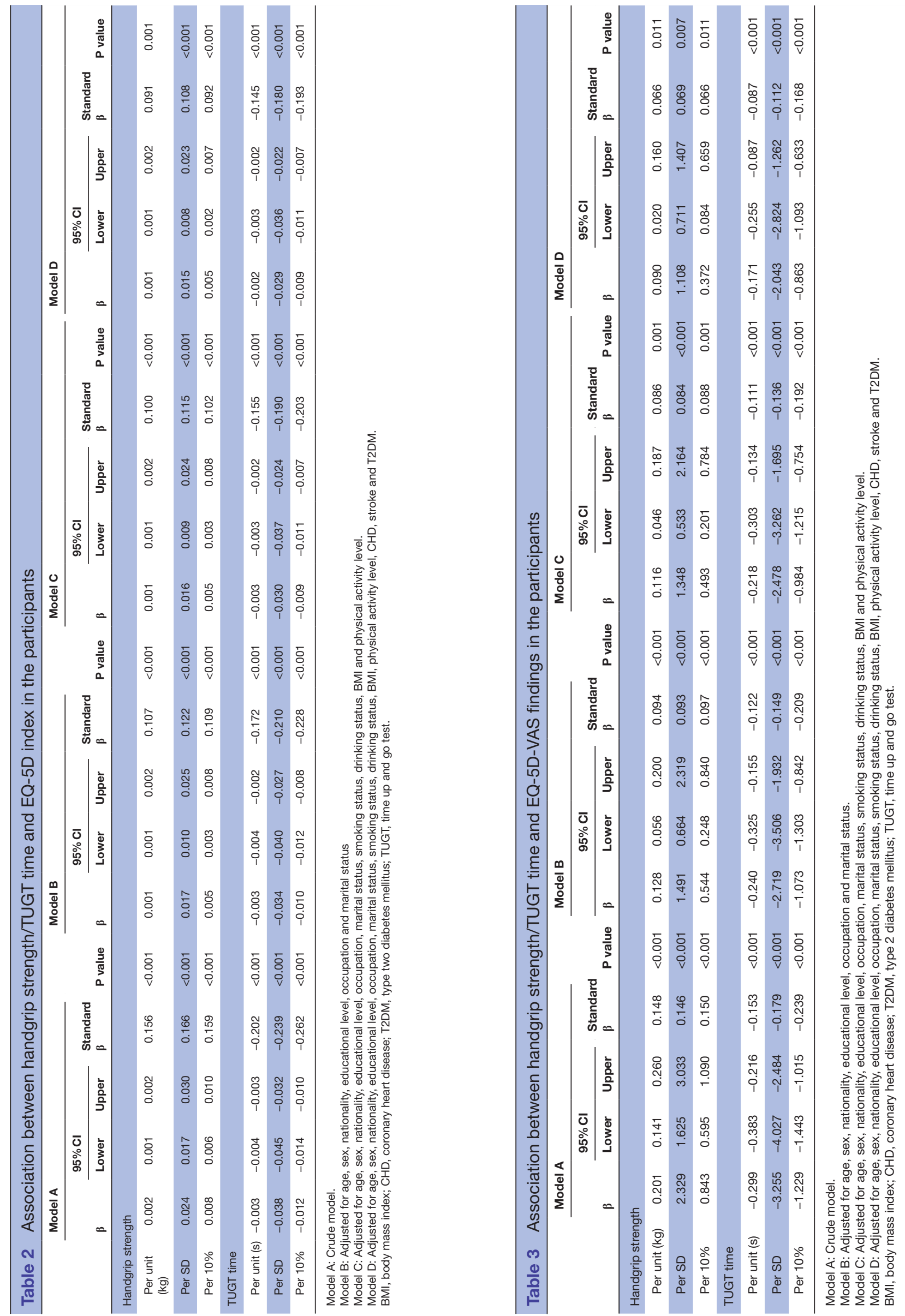


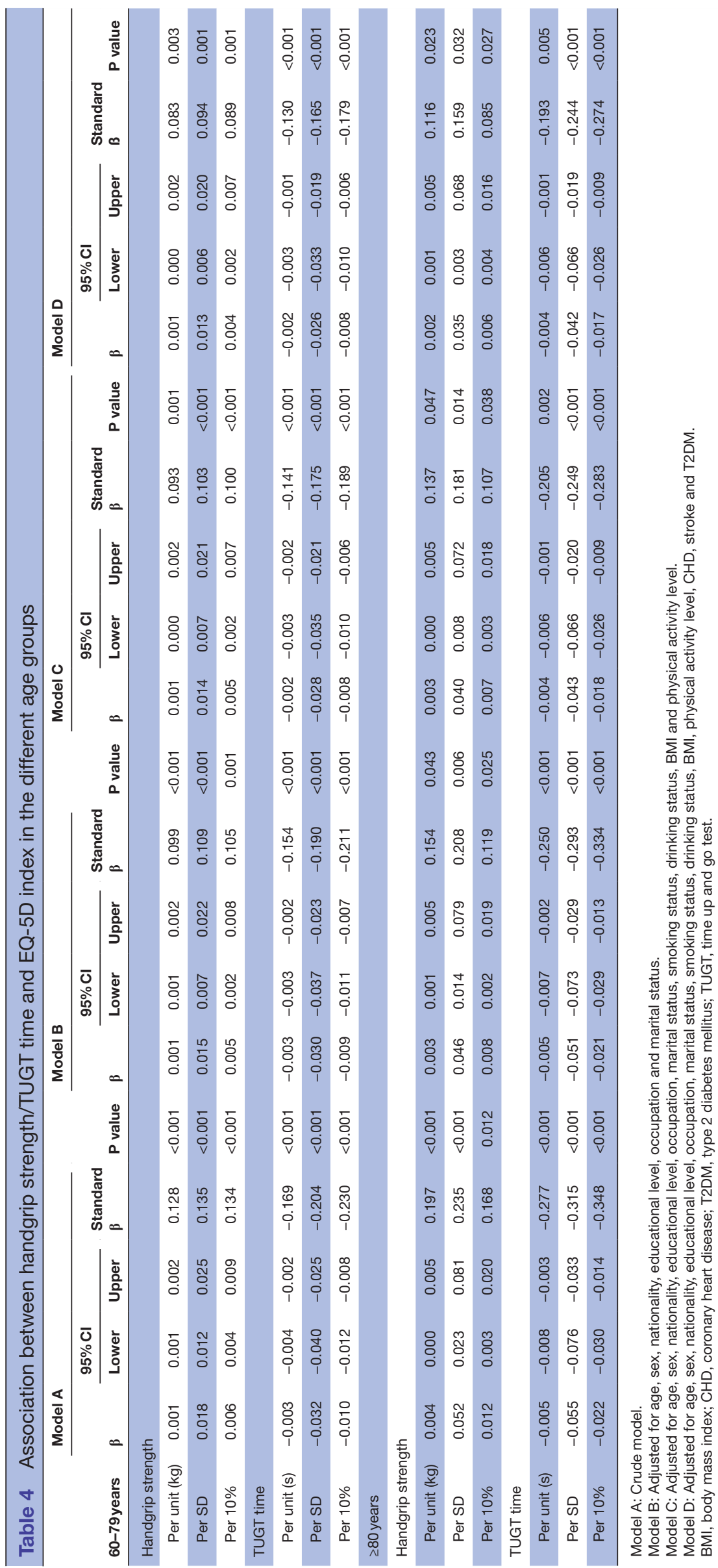




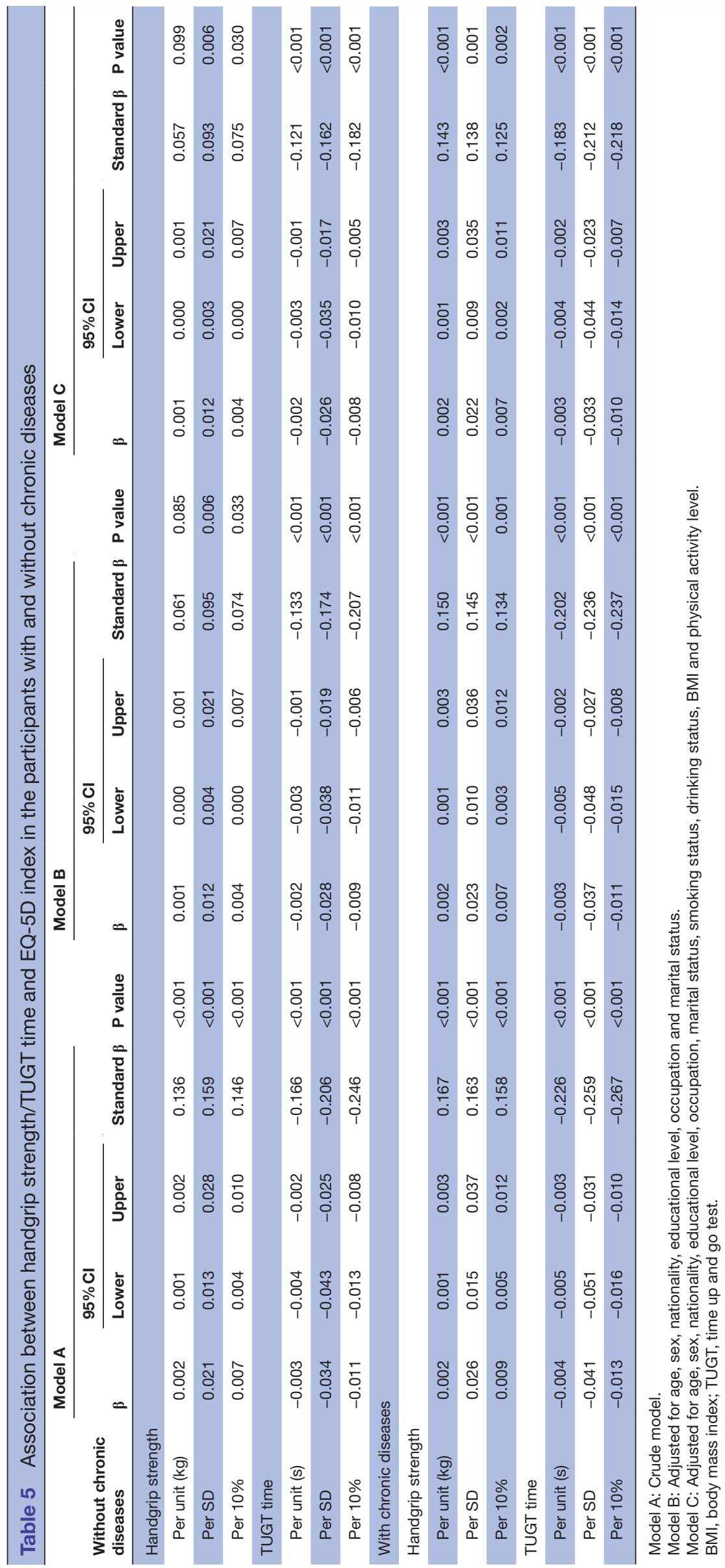



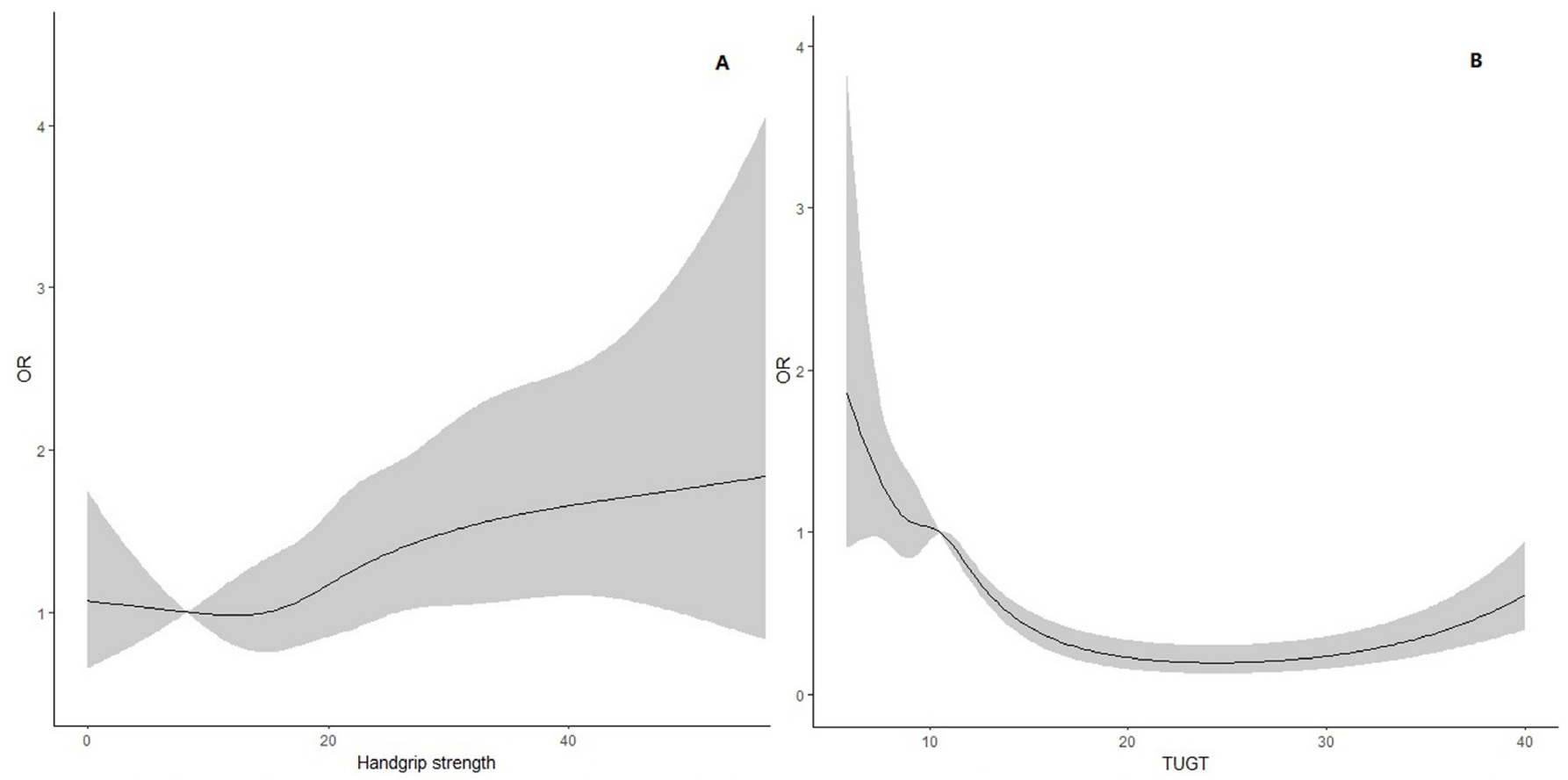

Figure 1 Smoothed effects of the handgrip strength (A) and TUGT time (B) using restricted cubic regression splines.

representative of the rural elderly population in China, as our participants resided, specifically, in a rural area on the outskirts of Beijing, which possesses a relatively high economic condition. Thus, care should be taken when generalising our results to the wider population. Third, participants' cognitive status is an important covariate. However, participants' cognitive statuses, in this study, were not collected separately, so an adjustment for this in the models used could not be made. Fourth, the measurement of physical activity used by our study was self-reported, due to the limitations of the participants' age and investigation conditions and, as a result, we could not use a more objective investigation tool. The TUGT was used to test the basic mobility skills of frail elderly persons, and the evaluation of their lower limb muscle strength may not be accurate as a result. Fifth, the economic issue is also an important covariable within this study, but we could not collect the participants' economic information directly. However, we adjusted for their educational levels and occupations instead, as measures of their overall economic status. ${ }^{29}$ Finally, owing to the inherent bias of cross-sectional studies, we could not conclude if there is a causal correlation between muscle strength and HRQoL among the participants. The results of the study need to be validated by a future interventional community research study.

The results of this study suggest a significant relationship between muscle strength (measured via handgrip strength and TUGT time) and HRQoL (measured via EQ-5D index and VAS score) in the Chinese rural elderly population. The functional forms of the participants' handgrip strength and TUGT time were different. The relationship was stronger in the older population (aged $\geq 80$ years), and for those with chronic diseases. The
HRQoL of the rural elderly population is an issue worthy of attention and, as a result, they should be advised to train and maintain adequate muscle strength, especially for individuals older than 80 years, and those with chronic diseases.

\section{Author affiliations}

${ }^{1}$ Institute of Geriatrics, Beijing Key Laboratory of Aging and Geriatrics, National Clinical Research Center for Geriatrics Disease, State Key Laboratory of Kidney Disease, the 2nd Medical Center, Chinese PLA General Hospital, Beijing, China ${ }^{2}$ Northern Military Area CDC, Shenyang, China

${ }^{3}$ The 2nd Medical Center, National Clinical Research Center for Geriatrics Disease, Chinese PLA General Hospital, Beijing, China

${ }^{4}$ Miyun County Hospital of Traditional Chinese Medicine, Beijing, China ${ }^{5}$ Department of Traditional Chinese Medicine and Acupuncture, The 2nd Medical Center, Chinese People's Liberation Army General Hospital, Beijing, China

Contributors SY, TL, HY and BJ contributed to data analysis and manuscript writing; SY, TL, HY, JW, ML, SW, YH and BJ contributed to study design and data collection; BJ and $\mathrm{HY}$ contributed to study design, manuscript revision and approval of final submission.

Funding This study was supported by the National Natural Science Foundation of China (81773502 and 81703308).

Competing interests We confirm that this manuscript contains original unpublished work and is not being submitted for publication elsewhere at the same time. This manuscript has been read and approved by all the authors; the requirements for authorship have been met; each author believes that the manuscript represents honest work.

Patient consent for publication Obtained.

Ethics approval The committee for medical ethics of the Chinese PLA General Hospital examined and approved our study (no. S2013-066-01).

Provenance and peer review Not commissioned; externally peer reviewed.

Data sharing statement All data relevant to the study are included in the article or uploaded as supplementary information.

Open access This is an open access article distributed in accordance with the Creative Commons Attribution Non Commercial (CC BY-NC 4.0) license, which permits others to distribute, remix, adapt, build upon this work non-commercially, 
and license their derivative works on different terms, provided the original work is properly cited, appropriate credit is given, any changes made indicated, and the use is non-commercial. See: http://creativecommons.org/licenses/by-nc/4.0/.

\section{REFERENCES}

1 Nations U. World population ageing 2013: Population Division of Department of Economic and Social Affairs New York, 2013. http:// www.un.org/en/development/desa/population/publications/pdf/ ageing/WorldPopulationAgeing2013.pdf.

2 Liu D, Darimont B. The health care system of the People's Republic of China: Between privatization and public health care. Int Soc Secur Rev 2013;66:97-116.

3 Zhou B, Chen K, Wang J, et al. Quality of Life and Related Factors in the Older Rural and Urban Chinese Populations in Zhejiang Province. Journal of Applied Gerontology 2011;30:199-225.

4 Organization WH. WHOQOL-BREF: introduction, administration, scoring and generic version of the assessment: field trial version, December 1996, 1996.

5 Kojima G, lliffe S, Jivraj S, et al. Association between frailty and quality of life among community-dwelling older people: a systematic review and meta-analysis. J Epidemiol Community Health 2016;70:716-21.

$6 \mathrm{Xu} \mathrm{H}$, Tang L, Hu Z, et al. Association between physical activity and health-related quality of life in elderly individuals with pre-diabetes in rural Hunan Province, China: a cross-sectional study. BMJ Open 2018;8:e019836.

7 Legrand D, Vaes B, Matheï C, et al. Muscle strength and physical performance as predictors of mortality, hospitalization, and disability in the oldest old. J Am Geriatr Soc 2014;62:1030-8.

8 Edwards MK, Loprinzi PD. Adequate Muscular Strength May Help to Reduce Risk of Residual-Specific Mortality: Findings From the National Health and Nutrition Examination Survey. J Phys Act Health 2018;15:369-73.

9 Fujita E, Takeshima N, Kato Y, et al. Effects of Body-weight Squat Training on Muscular Size, Strength and Balance Ability in Physically Frail Older Adults. International Journal of Sport and Health Science 2016;14:21-30.

10 Haider S, Luger E, Kapan A, et al. Associations between daily physical activity, handgrip strength, muscle mass, physical performance and quality of life in prefrail and frail communitydwelling older adults. Qual Life Res 2016;25:3129-38.

11 Chen H, Yu-Cheung W, Chan K. Social Capital among Older Chinese Adults: An Exploratory Study of Quality of Life and Social Capital in a Chinese Urban Community. The International Journal of Interdisciplinary Social Sciences: Annual Review 2009;4:107-24.

12 Krabbe P, Weijnen T. Guidelines for analysing and reporting EQ-5D outcomes. In: Brooks R, Rabin R, de Charro F, eds. The Measurement and Valuation of Health Status Using EQ-5D: A European Perspective: Evidence from the EuroQol BIOMED Research Programme. Springer Netherlands: Dordrecht, 2003:7-19.
13 Zhu YQ, Peng N, Zhou M. Effect of Tai Ji Quan Training on Strength and Function of Lower Limbs in the Aged]. Zhongguo Zhong xi yi jie he za zhi Zhongguo Zhongxiyi jiehe zazhi = Chinese journal of integrated traditional and Western medicine / Zhongguo Zhong xi yi jie he xue hui, Zhongguo Zhong yi yan jiu yuan zhu ban 2016;36:49.

14 Jung $\mathrm{H}$, Yamasaki M. Association of lower extremity range of motion and muscle strength with physical performance of communitydwelling older women. J Physiol Anthropol 2016;35:30.

15 Picone EN. The timed up and go test. Am J Nurs 2013;113:56-9.

16 Yq W, Liu K, Tang X, et al. [Empirical research of measuring elderly health utility in the outskirts of Beijing by using European quality of life 5-dimensions]. Journal of Peking University 2012;44:397.

17 Investigators WMPP. The World Health Organization MONICA Project (monitoring trends and determinants in cardiovascular disease): a major international collaboration. WHO MONICA Project Principal Investigators. J Clin Epidemiol 1988;41:105-14.

18 Power D. American Diabetes Association. Standards of medical care in diabetes--2006. Diabetes Care 2006;29 Suppl 1:S4-36.

19 Organization WH. Guidelines for the conduct of tobacco-smoking surveys among health professionals: report of a WHO meeting held in Winnipeg. Canada 1983:7-9.

20 Lam KB, Jiang CQ, Jordan RE, et al. Prior TB, smoking, and airflow obstruction: a cross-sectional analysis of the Guangzhou Biobank Cohort Study. Chest 2010;137:593-600.

21 ZY-gYQX L. Study on the Relationship between Health- related Quality of Life and Its Relevance from the Individual and Group Perspective. Chinese Health Economics 2018;37:77-80.

22 Whynes DK. TOMBOLA Group. Correspondence between EQ-5D health state classifications and EQ VAS scores. Health Qual Life Outcomes 2008;6:94.

23 Zhang L, Wang H, Wang L. Health Related Quality of Life in West Rural Population. Chinese Health Economics 2005;24:8-11.

24 Wang H, Kindig DA, Mullahy J. Variation in Chinese population health related quality of life: results from a EuroQol study in Beijing, China. Qual Life Res 2005;14:119-32.

25 Oh SH, Kim DK, Lee SU, et al. Association between exercise type and quality of life in a community-dwelling older people: A crosssectional study. PLoS One 2017;12:e0188335.

26 Taekema DG, Gussekloo J, Maier AB, et al. Handgrip strength as a predictor of functional, psychological and social health. A prospective population-based study among the oldest old. Age Ageing 2010;39:331-7.

27 Boyé ND, Mattace-Raso FU, Van Lieshout EM, et al. Physical performance and quality of life in single and recurrent fallers: data from the Improving Medication Prescribing to Reduce Risk of Falls study. Geriatr Gerontol Int 2015;15:350-5.

28 Sayer AA, Syddall HE, Martin HJ, et al. Is grip strength associated with health-related quality of life? Findings from the Hertfordshire Cohort Study. Age Ageing 2006;35:409-15.

29 de Meulemeester J-L, Rochat D. A causality analysis of the link between higher education and economic development. Econ Educ Rev 1995;14:351-61. 